\title{
Prevalence of fatty liver disease in patients with inflammatory bowel disease: a transient elastography study on the basis of a controlled attenuation parameter
}

\author{
Haluk Tarik KANI @ , Ilknur DELIKTAS @ , Yusuf YILMAZ
}

\begin{abstract}
Objective: Inflammatory bowel disease (IBD) is a chronic recurrent inflammatory disorder of the gastrointestinal tract which has an unknown etiology. In this study, we aimed to investigate the fatty liver disease frequency in IBD patients using transient elastography (TE) which is a non-invasive and accurate method for the diagnosis of liver fibrosis.

Material and Methods: The data were collected from registered patients retrospectively. On the basis of previous studies, a cut-off value of $238 \mathrm{~dB} / \mathrm{m}$ for controlled attenuation parameter (CAP) was used for the diagnosis of hepatic steatosis. In this study we have also settled CAP value above $238 \mathrm{~dB} / \mathrm{m}$ as fatty liver.

Results: A total of 99 patients were enrolled in the study. Mean age was $45.59 \pm 11.72$ years and $41(41.4 \%)$ patients were female. Fifty-eight patients (58.6\%) had Crohn's disease, 39 $(39.4 \%)$ had ulcerative colitis and $2(2.0 \%)$ had indeterminate colitis. In $8(8.1 \%)$ patients extra-large probe and in $91(91.9 \%)$ patients medium probe was used to detect liver steatosis. Fatty liver disease was detected in $44(44.4 \%)$ patients with IBD.

Conclusion: Despite the nutrition disorders in IBD, fatty liver disease was seen in $44.4 \%$ of patients in our cohort. However, fatty liver disease may be seen without any aminotransferase elevation in IBD patients. Therefore, liver steatosis should always be kept in mind and careful attention should be paid during follow-up of patients with IBD in our daily practice.
\end{abstract}

Keywords: Continued attenuation parameter, Inflammatory bowel disease, Non-alcoholic fatty liver disease

Haluk Tarik Kani $(\bowtie)$, Yusuf Yilmaz

Division of Gastroenterology, Department of Internal Medicine, School of Medicine, Marmara University Hospital, Pendik, Istanbul, Turkey and

Department of Gastroenterology, Health Sciences Institute, Marmara University, Maltepe, Istanbul, Turkey

e-mail:drhtkani@gmail.com

Ilknur Deliktas

Department of Internal Medicine, School of Medicine, Marmara University Hospital, Pendik, Istanbul, Turkey

Submitted: 11.11 .2018

Accepted: 28.12.2018

\section{Introduction}

Non-alcoholic fatty liver disease (NAFLD) is characterized by fat deposition in hepatocytes (over $5 \%$ of liver weight) in patients without a history of significant alcohol consumption and concurrent chronic liver disease [1,2]. Liver biopsy is the reference standard for NAFLD diagnosis, however, it is an invasive, complex and expensive method [3]. Fatty liver may be diagnosed by ultrasonography only when the presence of fat infiltration exceeds $30 \%$ in the liver. Therefore, hepatic steatosis may not always be detected by ultrasound [4]. Transient elastography (TE) is more sensitive than traditional liver USG in diagnosing fatty liver using controlled attenuation parameter (CAP). CAP is an indicator that shows the fat impedance in liver [5-11]. In a study, $238 \mathrm{~dB} / \mathrm{m}$ cut-off showed at least $10 \%$ steatosis with a $91 \%$ sensitivity and $81 \%$ specificity [5]. We also showed that TE detected fatty liver in $22.5 \%$ of patients who were not diagnosed as fatty liver with traditional USG in our previous study [10].

Non-alcoholic fatty liver disease is the most common etiology of elevated transaminases in inflammatory bowel disease (IBD) patients. NAFLD prevalence differed from $6.2 \%$ to $40 \%$ and liver fibrosis prevalence differed from $6.4 \%$ to $10 \%$ in the current literature [12-18]. All these previous studies diagnosed fatty liver disease using traditional USG but CAP value was not evaluated. In this study, we aim to investigate the hepatic steatosis frequency in IBD patients using CAP obtained via Fibroscan. 


\section{Materials and Methods}

The study was approved by the Marmara University, School of Medicine Ethics Committee (Approval Date: 02 March 2018, Approval Number: 09.2018.212).

The demographic data and CAP obtained via Fibroscan were retrospectively collected from the electronic files of the patients. The patients were admitted to our institute for evaluation by transient elastography without any specific indication from November 2012 to April 2017. Patients with significant alcohol consumption and concurrent chronic liver disease according to the electronic data were excluded from the study. IBD was diagnosed with the current European Crohn's and Colitis Organization guidelines at that time according to biochemical, endoscopic and pathological findings. Transient elastography examinations were performed with a Fibroscan 502 touch device (Echosens SA, Paris, France) following the manufacturer's instructions [10, 11]. Liver stiffness measurement (LSM) assessment was performed using medium (M) and extralarge (XL) probes. Procedures were performed on the right lobe of the liver through the intercostal area. The ratio of the interquartile range of the CAP to the median was calculated and considered as an indicator of variability. The final CAP value, ranging between 100 and $400 \mathrm{~dB} / \mathrm{m}[6-9,19]$, was the median value of individual measurements. On the basis of previous studies, a cut-off value of $238 \mathrm{~dB} / \mathrm{m}$ for CAP was used for the diagnosis of hepatic steatosis [5]. In this study we also settled CAP value above $238 \mathrm{~dB} / \mathrm{m}$ as fatty liver.

\section{Statistical Analysis}

Normally distributed data was expressed as mean \pm standard deviation, and the non-normal distributed data was expressed with the median values. Categorical data was compared with Chi-square test and $\mathrm{p}<0.05$ was accepted as significant.

\section{Results}

A total of 99 patients were enrolled in the study. Mean age was $45.59 \pm 11.72(25-72)$ years. Forty-one $(41.4 \%)$ patients were female and $58(58.6 \%)$ patients were male. Fifty-eight patients $(58.6 \%)$ had Crohn's disease (CD), 39 (39.4\%) had ulcerative colitis (UC) and $2(2.0 \%)$ had indeterminate colitis. XL probe was used in $8(8.1 \%)$ patients and $\mathrm{M}$ probe was used in $91(91.9 \%)$ patients to detect the liver steatosis. Mean CAP value was $236.56 \pm 61.21(100$
- 390) $\mathrm{kPa}$ and NAFLD was detected in 44 (44.4\%) patients using Fibroscan. Besides, NAFLD was detected in $48.3 \%$ $(n=28), C D$ in $38.5 \%(n=15)$ and $U C$ respectively and this difference was not statistically significant.

\section{Discussion}

The prevalence of NAFLD in IBD patients based on transient elastography was $44.4 \%$ in our study. Several studies found the prevalence of NAFLD in IBD patients between $6.2 \%$ and $40 \%$ [13-15]. To the best of our knowledge, this study is the first one that measured CAP in patients with hepatic steatosis using transient elastography. According to current literature, five studies diagnosed the fatty liver disease on classical USG scans [13, 15, 20-22], one study detected the disease using USG, computer tomography or magnetic resonance imaging [14] and one study detected the disease by hepatic steatosis index and laboratory results without using any imaging modality [23]. CAP is a sensitive diagnostic method for detecting fatty liver and our study indicated the highest NAFLD prevalence when compared with current literature.The prevalence variability among previous studies may be related to the type of diagnostic imaging modalities. Also, NAFLD prevalence between $8.9 \%$ and $39.5 \%$ was detected in CD patients and between $13.6 \%$ and $35.5 \%$ in UC patients in previous studies. These higher results may be attributable to the diagnostic accuracy of TE and CAP again.

There is no population-based NAFLD prevalence study in our country. In a previous study, NAFLD was shown to be $23.2 \%$ in healthy medical students [24]. Our study population was older and with chronic diseases and this may express the difference between the two studies.

Disclosure The authors declared no financial conflicts of interest.

\section{References}

1. Rinella M E. Nonalcoholic fatty liver disease: a systematic review. JAMA 2015;313:2263-73. doi:10.1001/ jama.2015.5370

2. EASL;EASD;EASO. Clinical Practice Guidelines for the management of non-alcoholic fatty liver disease. J Hepatol 2015;64:1388-402. doi:10.1016/j.jhep.2015.11.004

3. Rockey D C, Caldwell S H, Goodman Z D, et al. Liver biopsy. Hepatology 2009; 49: 1017-44. doi:10.1002/hep.22742

4. Schwenzer N F, Springer F, Schraml C, Stefan N, Machann J, Schick F. Non-invasive assessment and quantification of liver 
steatosis by ultrasound, computed tomography and magnetic resonance. J Hepatol 2009;51:433-45. doi:10.1016/j. jhep.2009.05.023

5. Sasso M, Beaugrand M, de Ledinghen V, et al. Controlled attenuation parameter (CAP): a novel VCTE guided ultrasonic attenuation measurement for the evaluation of hepatic steatosis: preliminary study and validation in a cohort of patients with chronic liver disease from various causes. Ultrasound Med Biol 2010; 36: 1825-35. doi:10.1016/j. ultrasmedbio.2010.07.005

6. Sasso M, Miette V, Sandrin L, et al. The controlled attenuation parameter (CAP): a novel tool for the non-invasive evaluation of steatosis using Fibroscan. Clin Res Hepatol Gastroenterol 2012;36: 13-20. doi:10.1016/j.clinre.2011.08.001

7. Sasso M, Tengher-Barna I, Ziol M, et al. Novel controlled attenuation parameter for noninvasive assessment of steatosis using Fibroscan $((\mathrm{R}))$ : validation in chronic hepatitis C. J Viral Hepat 2012;19:244-53. doi:10.1111/j.13652893.2011.01534.x

8. de Ledinghen, V, Vergniol J, Foucher J, et al. Non-invasive diagnosis of liver steatosis using controlled attenuation parameter (CAP) and transient elastography. Liver Int 2012; 32:911-8. doi:10.1111/j.1478-3231.2012.02820.x

9. Boursier J, Cales P. Controlled attenuation parameter (CAP): a new device for fast evaluation of liver fat? Liver Int 2012;32:875-7. doi:10.1111/j.1478-3231.2012.02824.x

10. Yilmaz Y, Ergelen R, Akin H, et al. Noninvasive detection of hepatic steatosis in patients without ultrasonographic evidence of fatty liver using the controlled attenuation parameter evaluated with transient elastography. Eur J Gastroenterol Hepatol 2013; 25:1330-34. doi:10.1097/ MEG.0b013e3283623a16

11. Yilmaz Y, Yesil A, Gerin F, et al. Detection of hepatic steatosis using the controlled attenuation parameter: a comparative study with liver biopsy. Scand J Gastroenterol 2014; 49: 6116. doi:10.3109/00365.521.2014.881548

12. Roman A L, Munoz F. Comorbidity in inflammatory bowel disease. World J Gastroenterol 2011;17: 2723-33. doi:10.3748/wjg.v17.i22.2723

13. Gisbert J P, Luna M, Gonzalez-Lama Y, et al. Liver injury in inflammatory bowel disease: long-term follow-up study of 786 patients. Inflamm Bowel Dis 2016;13:1106-14. doi:10.1002/ibd.20160

14. Sourianarayanane A, Garg G, Smith $T$ H, et al. Risk factors of non-alcoholic fatty liver disease in patients with inflammatory bowel disease. J Crohns Colitis 2013; 7: e27985. doi:10.1016/j.crohns.2012.10.015

15. Bargiggia S, Maconi G, Elli M, et al. Sonographic prevalence of liver steatosis and biliary tract stones in patients with inflammatory bowel disease: study of 511 subjects at a single center. J Clin Gastroenterol 2003;36: 417-20. doi: 10.1097/00004.836.200305000-00012

16. Barbero-Villares A, Mendoza Jimenez-Ridruejo J, Taxonera $\mathrm{C}$, et al. Evaluation of liver fibrosis by transient elastography (Fibroscan(R)) in patients with inflammatory bowel disease treated with methotrexate: a multicentric trial. Scand J Gastroenterol 2012;47:575-9. doi:10.3109/00365.521.2011. 647412

17. Thin L W, Lawrance I C, Spilsbury K, et al. Detection of liver injury in IBD using transient elastography. J Crohns Colitis 2014; 8: 671-7. doi:10.1016/j.crohns.2013.12.006

18. Chao C Y, Battat R, Al Khoury A, et al. Co-existence of nonalcoholic fatty liver disease and inflammatory bowel disease: A review article. World J Gastroenterol 2016;22:7727-34. doi:10.3748/wjg.v22.i34.7727

19. Myers R P, Pollett A, Kirsch R, et al. Controlled Attenuation Parameter (CAP): a noninvasive method for the detection of hepatic steatosis based on transient elastography. Liver Int 2012;32:902-10. doi:10.1111/j.1478-3231.2012.02781.x

20. de Fazio C, Torgano G, de Franchis R, et al. Detection of liver involvement in inflammatory bowel disease by abdominal ultrasound scan. Int J Clin Lab Res 1992;21:3147. doi:10.1007/bf02591669

21. Riegler G, D'Inca R, Sturniolo G C, et al. Hepatobiliary alterations in patients with inflammatory bowel disease: a multicenter study. Caprilli \& Gruppo Italiano Studio Colon-Retto. Scand J Gastroenterol 198;33:93-8. doi:10.1080/003.655.29850166275

22. Yamamoto-Furusho J K, Sanchez-Osorio M, Uribe M. Prevalence and factors associated with the presence of abnormal function liver tests in patients with ulcerative colitis. Ann Hepatol 2010;9:397-401.

23. Bessissow T, Le N H, Rollet K, et al. Incidence and predictors of nonalcoholic fatty liver disease by serum biomarkers in patients with inflammatory bowel disease. Inflamm Bowel Dis 2016;22:1937-44. doi:10.1097/mib.000.000.0000000832

24. Kaya E, Demir D, Alahdab Y O, et al. Prevalence of hepatic steatosis in apparently healthy medical students: a transient elastography study on the basis of a controlled attenuation parameter. Eur J Gastroenterol Hepatol 2016;28: 1264-7. doi:10.1097/meg.000.000.0000000681 